\title{
Theileria equi isolates vary in susceptibility to imidocarb dipropionate but demonstrate uniform in vitro susceptibility to a bumped kinase inhibitor
}

\author{
Siddra A Hines ${ }^{1 *}$, Joshua D Ramsay ${ }^{1}$, Lowell S Kappmeyer ${ }^{1,2}$, Audrey OT Lau', Kayode K Ojo3 \\ Wesley C Van Voorhis ${ }^{3}$, Donald P Knowles ${ }^{1,2}$ and Robert H Mealey ${ }^{1 *}$
}

\begin{abstract}
Background: The apicomplexan hemoparasite Theileria equi is a causative agent of equine piroplasmosis, eradicated from the United States in 1988. However, recent outbreaks have sparked renewed interest in treatment options for infected horses. Imidocarb dipropionate is the current drug of choice, however variation in clinical response to therapy has been observed.

Methods: We quantified the in vitro susceptibility of two T. equi isolates and a lab generated variant to both imidocarb dipropionate and a bumped kinase inhibitor compound 1294. We also evaluated the capacity of in vitro imidocarb dipropionate exposure to decrease susceptibility to that drug. The efficacy of imidocarb dipropionate for clearing infection in four T. equi infected ponies was also assessed.

Results: We observed an almost four-fold difference in imidocarb dipropionate susceptibility between two distinct isolates of T. equi. Four ponies infected with the less susceptible USDA Florida strain failed to clear the parasite despite two rounds of treatment. Importantly, a further 15 -fold decrease in susceptibility was produced in this strain by continuous in vitro imidocarb dipropionate exposure. Despite a demonstrated difference in imidocarb dipropionate susceptibility, there was no difference in the susceptibility of two T. equi isolates to bumped kinase inhibitor 1294.

Conclusions: The observed variation in imidocarb dipropionate susceptibility, further reduction in susceptibility caused by drug exposure in vitro, and failure to clear T. equi infection in vivo, raises concern for the emergence of drug resistance in clinical cases undergoing treatment. Bumped kinase inhibitors may be effective as alternative drugs for the treatment of resistant $T$. equi parasites.
\end{abstract}

Keywords: Theileria equi, Equine piroplasmosis, Imidocarb dipropionate, Apicomplexan, Bumped kinase inhibitor, Drug susceptibility

\section{Background}

Theileria equi, one of the two causative agents of equine piroplasmosis, is a tick-transmitted hemoprotozoan parasite classified within the phylum Apicomplexa. The United States has been considered free of equine piroplasmosis for several decades, however the occurrence of multiple U.S. outbreaks in recent years has caused a resurgence of interest in effective treatment options. Infection can be clinical or subclinical, but persists even

\footnotetext{
*Correspondence: siddra@vetmed.wsu.edu; rhm@vetmed.wsu.edu 'Department of Veterinary Microbiology and Pathology, College of Veterinary Medicine, Washington State University, Pullman, WA 99164-7040, USA Full list of author information is available at the end of the article
}

with resolution of clinical signs [1-3]. Strict federal regulations for the elimination of infected horses have been established in an attempt to maintain piroplasmosis-free status in the U.S., requiring T. equi positive horses to be euthanized, permanently quarantined, exported to the country of origin, or treated under the current USDAARS-APHIS treatment program $[4,5]$. This program is currently the only federally-sanctioned option for treatment in the U.S., as full elimination of parasites from the host must be verified in order for treated horses to no longer be considered potential reservoirs of infection [5].

For most apicomplexan parasitic pathogens, the goal of treatment is to minimize the clinical impact of disease. 
Complete elimination of this type of pathogen is a considerable challenge, particularly with organisms such as $T$. equi which causes persistent infection [3]. Imidocarb dipropionate (IMD) is a dicationic diamidine of the carbanilide series of antiprotozoal compounds, and is the drug most commonly used to treat equine piroplasmosis caused by both $T$. equi and Babesia caballi. In a recent major outbreak localized in Texas [6], IMD successfully cleared over 163 naturally infected horses of T. equi (Dr. Angela Pelzel, USDA-APHIS, personal communication) $[4,5]$. However, variation in response to treatment with an identical IMD protocol has been observed in both natural and experimental T. equi infection [4,7-10], with treatment failure characterized by parasite persistence and recrudescence of parasitemia following discontinuation of treatment. The identification of drug resistance in other apicomplexan parasites [11-13] indicates drug resistance is likely an important factor in T. equi treatment failures. In particular, the human malarial agent Plasmodium falciparum has exhibited continuously evolving multidrug resistance, necessitating continued development of novel antimalarial drugs for effective treatment. Importantly, failure of treatment with previously effective drug protocols is almost invariably associated with decreased in vitro susceptibility to the treatment drug $[11,14]$.

Many drugs have been assessed in vitro for efficacy against $T$. equi [15-21]; however a large number of these are not biologically relevant or feasible for use in horses. Although IMD is commonly used clinically, susceptibility has never been evaluated in vitro for $T$. equi nor compared between parasite strains. Importantly, the potential impact of IMD exposure on the susceptibility of T. equi to this drug, a known factor in the development of drug resistance in many other organisms [14,22-27], has not been investigated.

Given the scarcity of treatment options for T. equi and the potential for drug resistance, evaluation of alternative and novel drugs is necessary. Bumped kinase inhibitors (BKIs) are a group of experimental compounds currently being investigated for in vitro and in vivo efficacy against malaria [28,29], toxoplasmosis [30,31], cryptosporidiosis [31,32], and other protozoal diseases [33]. The BKIs selectively inhibit apicomplexan calcium-dependent protein kinases (CDPKs), which are critical for multiple parasitic physiological functions including parasite motility and invasion as well as in secretory pathways and replication [28]. Importantly, these CDPKs are absent in vertebrates, making them excellent anti-apicomplexan chemotherapeutic candidates [34]. Specifically, BKIs are competitive inhibitors of ATP-binding, and gatekeeper residue size appears to be a major factor in the selectivity of BKIs. These residues in apicomplexan CDPKs are small, typically glycine, serine, or threonine [28,34], which allow access to the ATP-binding pocket for BKIs to bind and inhibit apicomplexan CDPKs. Although CDPKs are not present in mammals, binding of most other mammalian kinases by BKIs is prevented by gatekeeper amino acid residues with large side chains that occlude access to the ATP-binding pocket. Therefore, the BKIs do not inhibit the proliferation of mammalian cells, and have been shown to be non-toxic in rodents [28,29,32].

In the present study, we evaluated the in vitro growth inhibitory effects of IMD against two isolates of T. equi, as well as a variant that was exposed in vitro to the drug. We also describe four ponies infected experimentally that failed to clear $T$. equi despite two rounds of IMD treatment following the established protocol $(4 \mathrm{mg} / \mathrm{kg}$, IM, q72 hrs for four doses) [7]. We then evaluated the in vitro efficacy of a novel bumped kinase inhibitor, BKI compound 1294, against two T. equi isolates with different degrees of susceptibility to IMD. This BKI compound was equally effective against both $T$. equi isolates, including the variant exposed in vitro to IMD. The results of this work should have implications in the design of therapeutic strategies against infections caused by drug-resistant $T$. equi.

\section{Methods}

\section{Chemical reagents}

For HL2A-FBS and -NHS culture media, HL-1 and HEPES were obtained from Fisher Scientific (Waltham, MA), HB101 supplement from Irvine Scientific (Santa Ana, CA), L-glutamine and AlbuMax from Gibco (Grand Island, NY) and penicillin/streptomycin and gentamicin from Sigma-Aldrich (St. Louis, MO). Hydroethidine was acquired from Invitrogen (Carlsbad, CA) as a $5 \mathrm{mM}$ solution solubilized in DMSO. Imidocarb dipropionate (Imizol ${ }^{\circledR}$ Merck, Millsboro, DE) at $344 \mathrm{mM}(120 \mathrm{mg} / \mathrm{mL})$ was utilized as the stock solution for all IMD assays and diluted in medium to reach experimental concentrations each day for use in the parasite growth inhibition assay described below. Bumped kinase inhibitor compound 1294 was generated as described [35], dissolved in DMSO at $20 \mathrm{mM}$, and then diluted in medium as above for use in the BKI 1294 growth inhibition assays.

\section{Evaluation of Theileria equi CDPK sequence}

Relevant amino acid sequences of BKI-binding CDPK proteins from other apicomplexans [33] including $P$. falciparum (PfCDPK1 [GI:124801388]), Babesia bovis (BbCDPK4 [GI: 154796736]), and T. gondii (TgCDPK1 [GI:255917998]) were obtained from GenBank and BLASTed (blastp, NCBI) against amino acid sequences predicted in the $T$. equi genome (GenBank accession number: ACOU00000000) in order to identify the T. equi ortholog. These sequences were then aligned and analyzed using ClustalW and BoxShade programs. 


\section{In vitro cultivation of Theileria equi}

The USDA Florida strain of T. equi (FL) [36,37] was obtained as a subculture from ongoing USDA research cultures. Cultures were initially grown in a microaerophilic environment $\left(5 \% \mathrm{O}_{2}\right)$ in modified HL2A-FBS medium [38] with $10 \mathrm{mM}$ hypoxanthine, $200 \mathrm{U} / \mathrm{mL}$ penicillin, and $200 \mu \mathrm{g} / \mathrm{mL}$ streptomycin added. Over time, the cultures were adapted to ambient $\mathrm{O}_{2}$ in a $5 \% \mathrm{CO}_{2} 37^{\circ} \mathrm{C}$ incubator and medium was converted from modified HL2A-FBS to modified HL2A-NHS (substituting normal horse serum for fetal bovine serum). Cultures were maintained in 24 well plates with an erythrocyte concentration of $10 \%$ in $1300 \mu \mathrm{L}$ total well volume and split 1:4 every other day, with $1000 \mu \mathrm{L}$ of medium changed on intervening days.

A novel T. equi isolate (TX) was obtained from a parasitemic horse as a part of a separate previous study [4] and adapted into culture. Briefly, an EDTA-anticoagulated whole blood sample was collected and centrifuged at 800 $g$ for 30 minutes. The plasma and leukocytes were discarded, and the erythrocytes washed three times in an equivalent volume of VYMs buffer, centrifuging as before. These infected erythrocytes were then placed in one well of a 24 well plate with $1000 \mu \mathrm{L}$ of media at a 1:1 ratio with uninfected normal horse erythrocytes ( $60 \mu \mathrm{L}$ of each). The plate was initially placed in a $37^{\circ} \mathrm{C}$ incubator with $5 \% \mathrm{O}_{2}$, $5 \% \mathrm{CO}_{2}$, and $90 \% \mathrm{~N}_{2}$ gas, however following successful culture initiation, TX was subsequently adapted to ambient $\mathrm{O}_{2}$ in a standard $5 \% \mathrm{CO}_{2}$ incubator and maintained as for the FL strain.

\section{In vitro parasite growth inhibition assay}

Initial IMD concentrations to be used in the assay were determined based on the pharmacokinetics of the drug in horses [39] and the in vitro susceptibility of other organisms to the drug $[22,40,41]$. To evaluate the dynamics of parasite growth during IMD exposure and to determine the appropriate incubation time for the assay, the FL strain was cultured in a 24-well plate for 72 hours with assessment of parasite growth via flow cytometry (below) and one mL medium change every 24 hours. Serial dilutions of IMD were tested in triplicate ranging from $1 \mathrm{nM}$ to $775 \mathrm{nM}$, with a target starting percent parasitized erythrocytes (PPE) for all wells of 0.25-0.5\%.

Based on these results, it was determined that the assay should be performed over 72 hours, starting at a low initial PPE. Further assays were performed in a 96-well plate, with a final erythrocyte concentration of $9 \%$ and target starting PPE of approximately $0.3 \%$. Two-fold serial dilutions of IMD ranged from $2.7 \mathrm{nM}$ to $344 \mathrm{nM}$, and triplicate samples for each concentration, including infected untreated controls, were evaluated. Triplicate wells of normal uninfected erythrocytes were also included as a control comparison. For assay initiation, 2x drug concentrations were prepared, with $100 \mu \mathrm{L}$ of the appropriate concentration placed into each well with $100 \mu \mathrm{L}$ of normal medium and $20 \mu \mathrm{L}$ of RBCs to reach the final $1 \times$ drug concentration for the assay and approximately $9 \% \mathrm{RBC}$ concentration in each well. At 24 and 48 hours, $160 \mu \mathrm{L}$ of medium containing the appropriate $1 \times$ drug concentration was changed in each well, with plain medium used for controls. The assay was then evaluated at 72 hours. Complete growth inhibition was considered to be achieved when the PPE remained at the starting level of $0.3-0.5 \%$ after 72 hours, indicating no active parasite growth.

The chemical structure of BKI 1294 has been previously published [30]. Drug concentrations tested for BKI 1294 included $0.3 \mu \mathrm{M}, 3 \mu \mathrm{M}, 6 \mu \mathrm{M}, 9 \mu \mathrm{M}, 12 \mu \mathrm{M}$, and $30 \mu \mathrm{M}$. Assay procedure was otherwise identical to that for IMD.

\section{Flow cytometric evaluation of parasite growth}

After 72 hours, all samples were transferred to a 96 well V-bottom plate to be stained with hydroethidine. Cells were centrifuged at $500 \mathrm{~g}$ at room temperature, and the culture medium supernatant discarded. They were then washed in $150 \mu \mathrm{L}$ of $1 \times$ PBS per well and centrifuged as before. Stock $5 \mathrm{mM}$ hydroethidine in anhydrous DMSO was diluted to $20 \mu \mathrm{g} / \mathrm{mL}$ in $1 \times \mathrm{PBS}$ and added at to all samples (including normal erythrocytes) at $100 \mu \mathrm{L}$ per well. Samples were incubated for 15 minutes at $37^{\circ} \mathrm{C}$ in $5 \% \mathrm{CO}_{2}$ in the absence of light, and $100 \mu \mathrm{L}$ of plain $1 \times$ PBS was added at the conclusion of the incubation prior to centrifugation. After removal of the supernatant, the cells were resuspended in $200 \mu \mathrm{L}$ of $1 \times$ PBS. Fifty $\mu \mathrm{L}$ of each sample was then diluted into approximately two $\mathrm{mL}$ of $1 \times$ PBS containing $0.2 \%$ sodium azide for flow cytometric analysis.

Cell suspensions were evaluated using a FACSCaliber flow cytometer equipped with CellQuest computer software (Becton Dickinson Immunocytometry Systems, San Jose, CA) on a Macintosh computer. The inclusion gate was based on the forward and side scatter features of uninfected erythrocytes stained with hydroethidine, and 50,000-150,000 events per sample were collected. Ethidium bromide fluoresces in the FL-2 channel, therefore argonlaser fluorescence excitation at $488 \mathrm{~nm}$ and emission at $585 \mathrm{~nm}$ (range 563-607 nm) were used for analysis in log Fl 2 data mode. Fluorescent profiles were recorded for later analysis with FCS Express software (De Novo software, Los Angeles, CA). Quadrant gating of generated dot plots (Fl-2 vs. side scatter) was based on stained uninfected erythrocyte controls to delineate between infected and uninfected cell populations.

\section{Determination of $\mathrm{IC}_{50}$ and $I \mathrm{C}_{90}$ values}

The PPE of each well was determined based on the percentage of the cell population characterized as RBCs that 
exhibited FL-2 fluorescence. Mean PPE for each drug concentration and controls was calculated by averaging the PPE of all three triplicate wells. Individual PPE values that deviated from the other replicates by $>0.5 \%$ were considered outliers and eliminated from data evaluation. Percent of maximum PPE was then calculated for each concentration using the mean PPE value in comparison to the highest PPE obtained in the assay for a given isolate. The 50\% ( $\left.\mathrm{IC}_{50}\right)$ and $90 \%\left(\mathrm{IC}_{90}\right)$ inhibitory concentrations were determined by fitting the curves with nonlinear regression using GraphPad Prism version 6.03 for Windows (GraphPad Software, La Jolla, CA).

\section{Exposure of Theileria equi parasites to imidocarb dipropionate in vitro}

Exposure of the FL strain of $T$. equi to IMD was performed via two different methods. The first method involved pulse exposure, with parasites exposed at 28.7 $\mathrm{nM}$ (the approximate $\mathrm{IC}_{50}$ value determined previously) for an initial period of 11 days, until growth had declined to a PPE of less than 1.5\%. Drug pressure was then removed and the parasites were allowed to recover for five days. They were then re-exposed to $28.7 \mathrm{nM}$ for 24 hours. Subsequent 24 hour re-exposures of the pulse exposure variant to increasing concentrations of IMD were performed over the next two months, allowing sufficient time for recovery between exposures, with the final re-exposure at $172 \mathrm{nM}$ (FL Exp variant 1). The third method of FL IMD exposure involved continuous exposure to increasing concentrations of IMD, starting at the lowest previously determined $\mathrm{IC}_{50}$ of the strain (24 nM) and gradually increasing up to $115 \mathrm{nM}$ (FL Exp variant 2).

The TX isolate was exposed continuously to $3.2 \mathrm{nM}$ IMD for 24 days and also to a higher concentration (9.5 nM IMD) for a short period of time (11 days).

\section{Infection and treatment of ponies with Theileria equi}

Four naïve mixed-breed ponies, 1-2 years of age, were experimentally infected with the USDA FL strain of $T$. equi via Rhipicephalus microplus tick transmission as a part of a separate research study, using previously published methods [42]. All ponies were confirmed positive for T. equi by nested PCR [4] and were initially treated with IMD ( $4 \mathrm{mg} / \mathrm{kg}$, IM, q72 hrs for four doses) at seven months post-infection. Post-treatment infection status was determined using nested PCR and cELISA [43]. Because this initial treatment failed to clear T. equi in each of the four ponies, the same treatment regimen was repeated 18 months post-infection and the same follow-up evaluations performed. All experiments involving animals were carried out in accordance with the recommendations in the Guide for the Care and Use of Laboratory Animals of the National Institutes of Health and in conformance with the United States Department of Agriculture animal research guidelines, under a protocol approved by the Washington State University Institutional Animal Care and Use Committee (ASAF \# 04163).

\section{Results}

Imidocarb dipropionate treatment of ponies experimentally infected with the USDA FL strain of Theileria equi

Sixteen days following the first round of IMD treatment (seven months post-infection), all four ponies appeared to be negative for $T$. equi based on nested PCR (Figure 1a). However, nested PCR was repeated at 17 months post-infection and all ponies were found to be positive. After the second round of IMD treatment at 18 months post-infection, the ponies again appeared negative for $T$. equi based on nested PCR performed 16 days post-treatment. However, re-evaluation 37 and 80 days (Figure $1 \mathrm{~b}$ ) after this second round of treatment (19-20 months post-infection) confirmed that all ponies again reverted to a positive state and that two rounds of IMD treatment failed to clear infection. Seropositivity was also confirmed at 14 and 20 months post-infection by cELISA (data not shown).

\section{In vitro susceptibility of Theileria equi to imidocarb propionate}

Development of the in vitro parasite growth inhibition assay using IMD for T. equi was successfully accomplished using the FL parasite strain, with evaluation of the PPE for each sample using flow cytometry (Figure 2). Regardless of the concentration of IMD, the PPE in all wells doubled or tripled over the first 24 hours. By 48 hours, the growth dynamics across IMD concentrations began to diverge, with parasite growth at higher concentrations leveling off and that at lower concentrations continuing to increase until reaching a maximum of $7-8 \%$ PPE at 72 hours. Overall, the final PPE across the range of drug concentrations reflected a dosedependent effect of IMD, with an $\mathrm{IC}_{50}$ of $24 \mathrm{nM}$ (Figure 2a). An $\mathrm{IC}_{90}$ could not be determined for this strain, because even at the maximum drug concentration $(775 \mathrm{nM})$ the PPE still reached $1.2 \%$, equivalent to $15.4 \%$ of the growth of untreated parasites. This indicated that the FL strain parasite population more than doubled from the starting PPE of $0.5 \%$ even at high IMD concentrations, reflecting a lack of complete growth inhibition.

The TX isolate originating from the 2009 outbreak $[4,6]$ was consistently more susceptible to IMD than the FL strain, with an $\mathrm{IC}_{50}$ of $6.4 \mathrm{nM}$; almost six-fold lower than FL (Figure 3a). In addition, an $\mathrm{IC}_{90}$ of $26 \mathrm{nM}$ could be determined for the TX isolate, as it did not actively 
a)

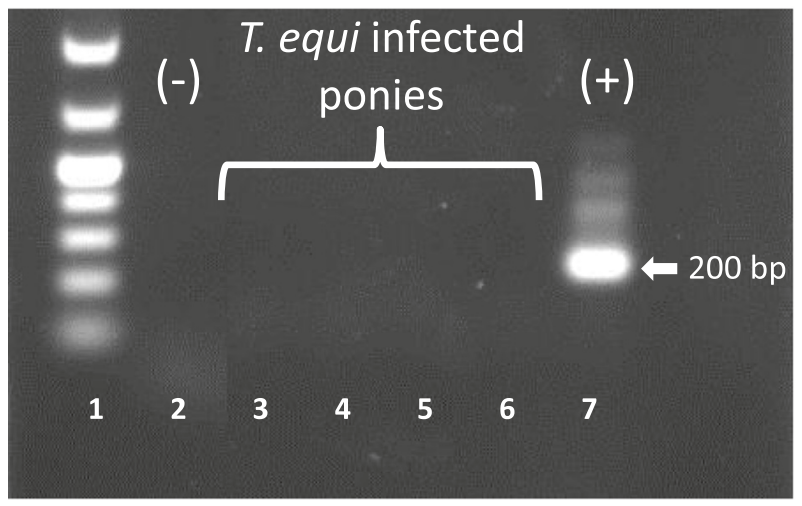

b)

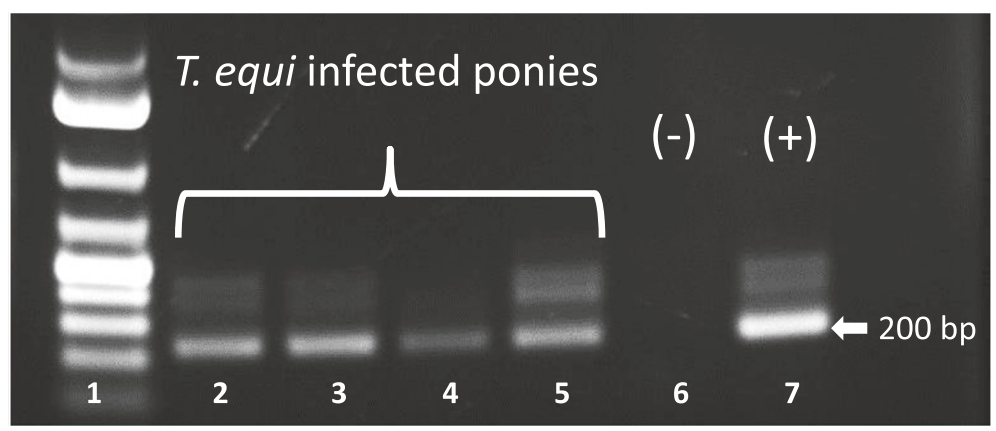

Figure 1 Nested PCR detection of $\boldsymbol{T}$. equi infection in ponies after failed IMD treatment. (a) 2\% agarose gel showing results from packed erythrocytes collected 16 days after the second round of IMD treatment. Lane 2: negative control erythrocytes from an uninfected horse (-). Lanes 3, 4, 5, 6: erythrocytes from the four IMD treated ponies. Lane 7: positive control erythrocytes from a known infected horse showing the 200 bp T. equi ema-1 amplicon (+). (b) T. equi ema-1 amplicons from packed erythrocytes collected 80 days after the second round of IMD treatment. Lanes 2, 3, 4, 5: erythrocytes from the four IMD treated ponies. Lane 6: negative control (-). Lane 7: positive control (+).

grow at higher drug concentrations, with PPE remaining at the starting level of $0.5 \%$.

\section{Imidocarb dipropionate exposure of Theileria equi and the effect on $I_{50}$}

Continuous exposure of the FL strain to increasing concentrations of IMD (up to $115 \mathrm{nM}$ ) increased the $\mathrm{IC}_{50}$ a maximum of 15 -fold in FL Exp variant 2 to $414 \mathrm{nM}$ (Figure 3b). Pulse exposure with IMD (up to $172 \mathrm{nM}$ ) increased the $\mathrm{IC}_{50}$ in FL Exp variant 1 to a lesser degree, approaching four-fold at $94 \mathrm{nM}$ (Figure 3b). However, this effect could not be duplicated in the TX isolate. Despite repeated attempts to expose TX to IMD, the $\mathrm{IC}_{50}$ remained the same at approximately $6 \mathrm{nM}$. Exposure of TX to a higher concentration of IMD (9.5 nM) resulted in irrevocable decline in culture PPE and eventual death of all exposed parasites.

\section{Gatekeeper residue of Theileria equi calcium-dependent protein kinase}

Due to the variability in response to IMD, alternative drugs for treatment of T. equi were considered. The BKI compounds were of interest, and therefore evaluation of the T. equi transcriptome for an appropriate CDPK drug target was undertaken. Amino acid sequence comparisons of the BKI-binding CDPKs in P. falciparum, $B$. bovis, and $T$. gondii against the $T$. equi predicted transcriptome identified the same protein kinase domaincontaining protein (GI: 510911326) as the putative CDPK ortholog in T. equi. Alignment of these four sequences revealed a threonine gatekeeper residue in T. equi, consistent with the small amino acid gatekeeper residues present in comparison organisms (Figure 4), all of which are susceptible to BKI compounds [33].

\section{In vitro susceptibility of Theileria equi to the bumped kinase inhibitor compound 1294}

For BKI 1294, the $\mathrm{IC}_{50}$ of the FL Exp variant 1 and the TX isolate were similar $(4.212 \mu \mathrm{M}$ and $3.887 \mu \mathrm{M}$ respectively) (Figure 5). Active parasite growth of both isolates was completely inhibited at the highest drug concentrations, with PPE limited to the starting level of $0.3 \%$ (3.5-3.6\% of the maximum PPE). Therefore, the $\mathrm{IC}_{90}$ was 


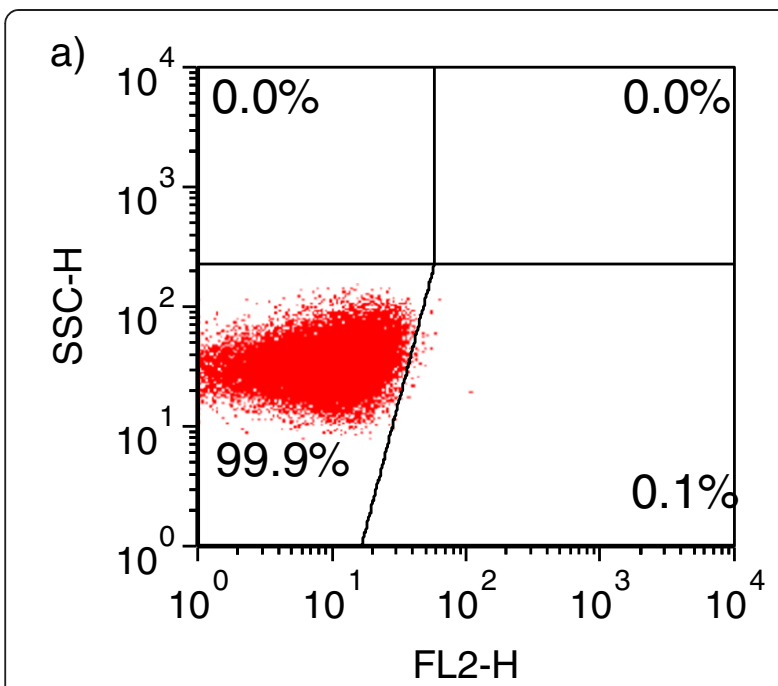

b)

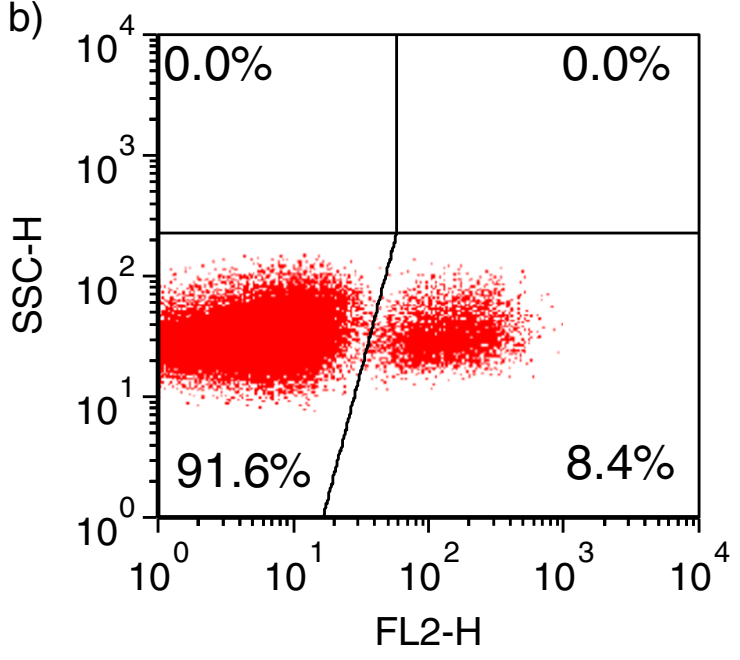

c)

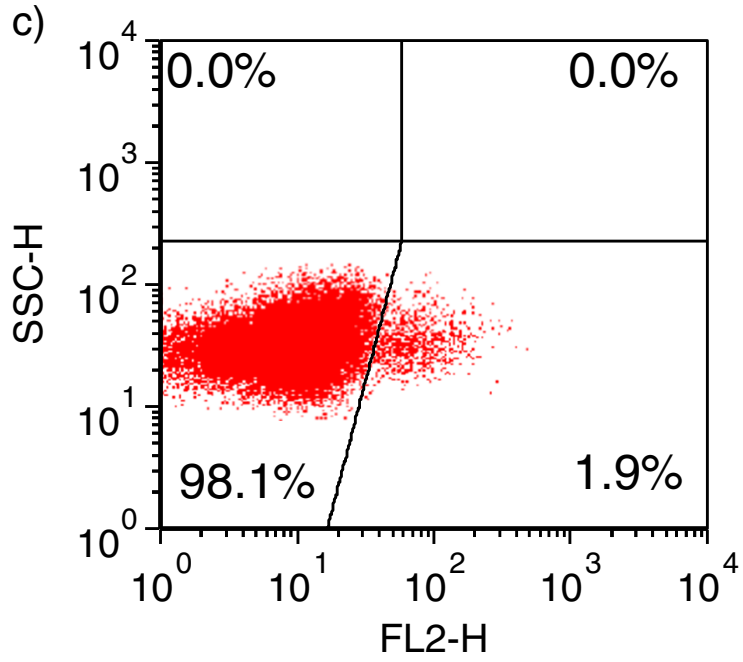

Figure 2 Flow cytometric scatter plots depicting percent parasitized erythrocytes (PPE). Representative samples from a 72 hour growth inhibition assay. Parasitized erythrocytes stained with hydroethidine were detected in the FL-2 channel, and are represented in the bottom right quadrant. (a) Normal uninfected erythrocytes stained with hydroethidine (negative control). (b) T. equi-infected erythrocytes incubated without imidocarb dipropionate (IMD; positive control). (c) T. equi-infected erythrocytes incubated with $2.76 \mu \mathrm{M} \mathrm{IMD}$.

achieved for BKI 1294 for both FL Exp variant 1 and TX (11.502 $\mu \mathrm{M}$ and $10.964 \mu \mathrm{M}$, respectively).

\section{Discussion}

Although the life cycle of T. equi in horses is biphasic, with an early pre-erythrocytic schizogony within leukocytes, this schizont stage is transient and does not appear to play a role in persistent infection of horses [42]. Instead, long term infection is perpetuated through infection and proliferation of merozoites in erythrocytes, making this merozoite stage most relevant for chemotherapeutic intervention. Therefore, erythrocyte cultures of merozoites were used in this study to evaluate in vitro susceptibility. The observed difference in IMD susceptibility between isolates, with the IMD $\mathrm{IC}_{50}$ of the $\mathrm{FL}$ strain almost six-fold higher than that of the TX isolate, was consistent with variation in efficacy that has been observed clinically. The overall success rate of IMD treatment for horses infected with the TX isolate during the 2009 outbreak was extremely high [4]. In contrast, the USDA FL strain has been used in multiple experimental settings to evaluate the efficacy of IMD in vivo, with infected horses often failing to clear FL following treatment with a protocol identical to that used in the TX outbreak $[7,8]$. This situation was also observed in the current study, with four FL-infected ponies failing to clear infection despite two rounds of IMD treatment at $4 \mathrm{mg} / \mathrm{kg}$ every 72 hours for 4 doses per round. Interestingly, the ponies appeared negative immediately after treatment, with parasite growth suppressed by IMD to the point that it could not initially be detected even with the extremely sensitive nested PCR method, which has been shown to detect a level of parasitemia of $0.000006 \%$ [44].

Previous research has demonstrated the presence of $T$. equi in the spleen of asymptomatic horses at times where it was undetectable in the peripheral blood via multiplex PCR [2]. Additionally, it is possible that the level of parasitemia was simply lower than the detectable limit, and that the small number of parasites surviving drug treatment was still sufficient to allow continued infection after a period of parasite recovery. The lack of complete in vitro inhibition of FL is consistent with the observed failure of IMD to clear the FL strain in the 

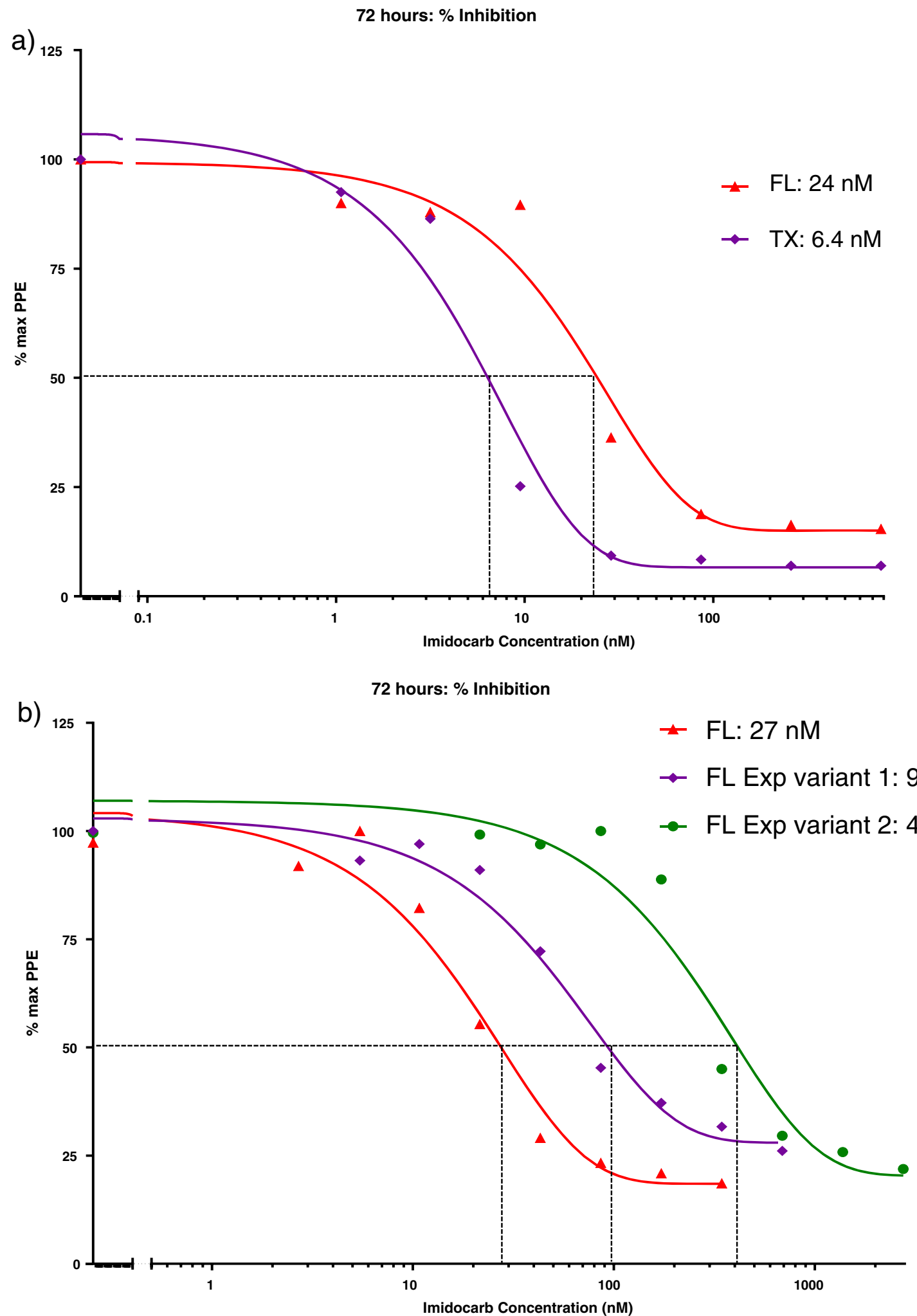

Figure 3 Variable in vitro susceptibility to IMD among different $T$. equi isolates. Each point on the nonlinear regression curve represents the mean percent of the maximum PPE determined in triplicate wells. $I_{50}$ is drug concentration resulting in $50 \%$ of the maximum PPE detected in wells not containing drug. (a) Susceptibility of the USDA FL strain (red) and the TX isolate (purple). (b) Susceptibility of FL Exp variant 1 (purple) and FL Exp variant 2 (green). 


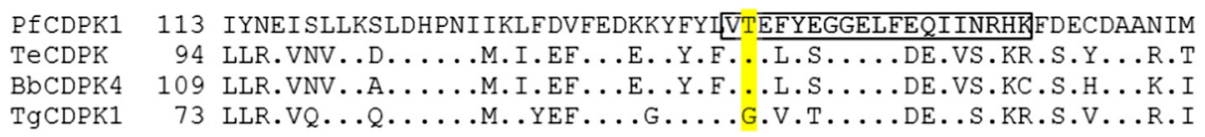

Figure 4 Amino acid alignment through the ATP-binding domains of calcium dependent protein kinases. Includes amino acid sequences from P. falciparum (Pf), T. equi (Te), B. bovis (Bb), and T. gondii (Tg). The ATP-binding region is boxed, and the gatekeeper residues are shaded.

infected ponies described in this study, as incomplete parasite inhibition led to a resurgence of parasitemia in vivo. This also raises further concern about the potential for development of resistance in these IMDexposed surviving parasites.

The pharmacokinetics of IMD administered intramuscularly in horses demonstrates a maximum plasma concentration of $0.2 \mu \mathrm{g} / \mathrm{mL}(574 \mathrm{nM})$ at a dose of $2.4 \mathrm{mg} / \mathrm{mL}$ [39]. Although this concentration greatly exceeds the $\mathrm{IC}_{50}$ of $24 \mathrm{nM}$ observed for the FL strain in the present study, this plasma concentration is maintained for only 2 hours in vivo and drops to undetectable levels (less than $0.0125 \mu \mathrm{g} / \mathrm{mL}$, or $36 \mathrm{nM}$ ) after 12 hours [39]. In contrast, the parasites in the present in vitro assay were exposed to IMD continuously at the tested concentrations for 72 hours, and in the case of FL, actively grew despite exposure to high concentrations of drug.

It has been postulated that a reservoir effect exists for IMD in vivo, as the drug is deposited in the liver and kidneys [45-48] as well as muscle in cattle [48] during the initial distribution phase. Trace amounts have been detected in the plasma of sheep up to four weeks after treatment [47]. This likely accounts for the overall efficacy of IMD for certain hemoprotozoan parasites, but makes it difficult to compare in vitro $\mathrm{IC}_{50}$ results with circulating IMD concentrations to determine if adequate drug concentrations are reached in the plasma. Additionally, the minimum detectable concentration of

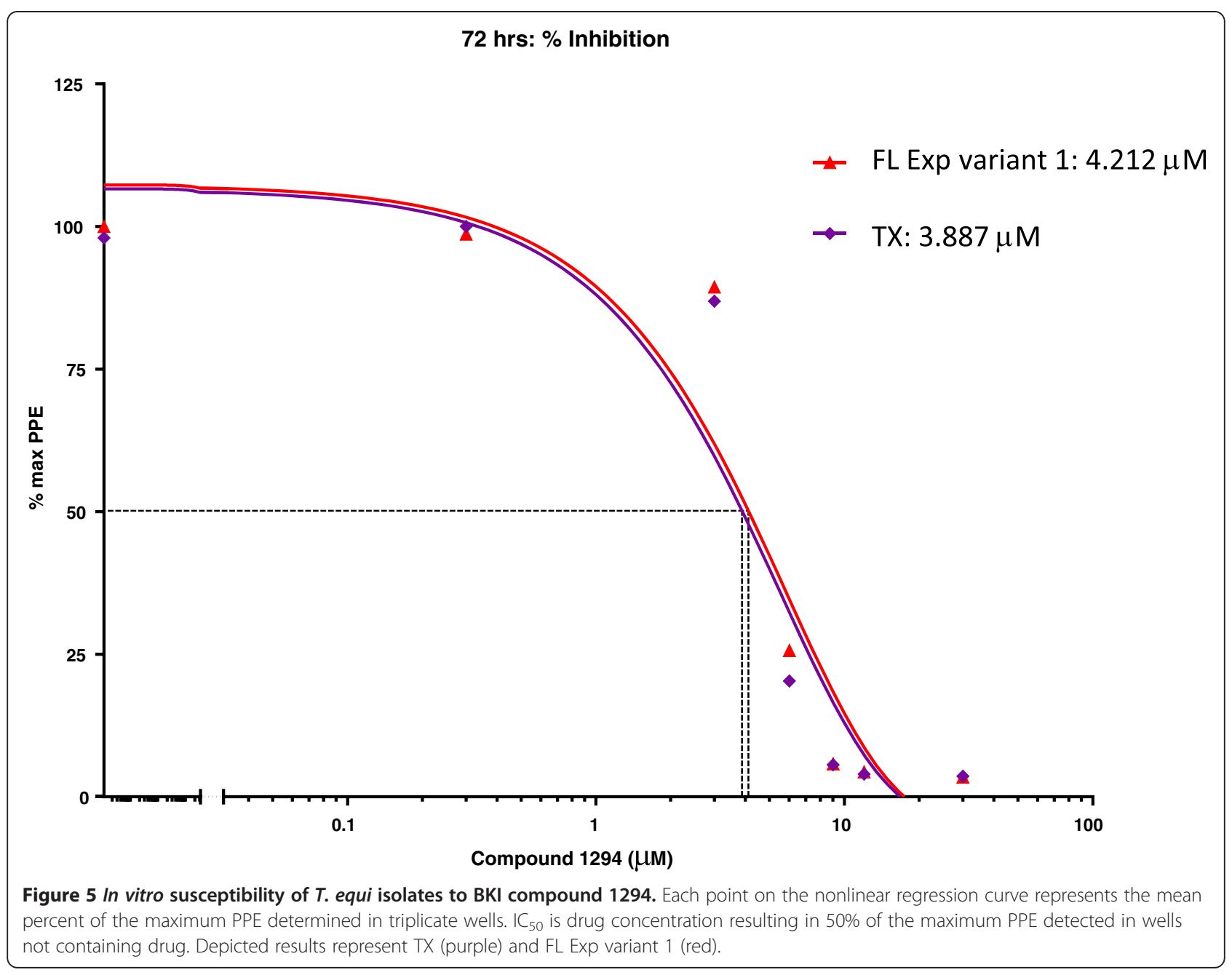


$0.0125 \mu \mathrm{g} / \mathrm{mL}(36 \mathrm{nM})$ for the HPLC assay utilized in the previous study [39] exceeded the $\mathrm{IC}_{50}$ of IMD for both FL and TX observed in the current study. Therefore, it is possible that the plasma concentration remains higher than the $\mathrm{IC}_{50}$ for a period longer than that suggested by the previous pharmacokinetics study. The $2.4 \mathrm{mg} / \mathrm{kg}$ dose used in that study was less than the $4 \mathrm{mg} / \mathrm{kg}$ dose currently recommended for clearance of T. equi $[4,7]$. Although the higher dose would be expected to achieve higher plasma concentrations, the fact that treatment failures occur at the higher dose indicates that IMD plasma concentrations above the $\mathrm{IC}_{50}$ are not always adequate for elimination of T. equi in vivo.

We observed that the $\mathrm{IC}_{50}$ for the FL strain could be further increased in vitro by exposure to IMD, as has been shown previously in vitro for B. bovis [22]. This finding suggests that resistance could emerge in natural parasite populations with exposure to IMD when animals are treated, particularly if complete inhibition of parasite growth cannot be achieved. As previously mentioned, trace amounts of IMD can be found in the plasma of sheep up to four weeks following treatment with IMD [47], which could result in prolonged drug selection pressure if the same occurs in horses. Although horses are not currently treated routinely in the United States, treatment occurs much more frequently in endemic countries. In these endemic countries the goal of therapy is to treat clinical disease rather than eliminate the parasite entirely from the host [49]. Therefore, treated horses can remain chronically infected and retain a population of parasites that was exposed to IMD yet was not killed by the drug. The possibility of developing IMD resistance is concerning, as there are very few options currently available for treatment of T. equi. In contrast to the observations with the FL strain however, IMD susceptibility in the TX isolate was not altered despite repeated IMD exposure. Thus, the capacity for drug exposure-associated changes in in vitro IMD susceptibility varies among T. equi strains. The underlying mechanism of IMD resistance among different $T$. equi strains is a focus of ongoing investigation.

The BKIs represent a novel class of compounds with potential as safe and effective treatment alternatives for T. equi infection, particularly for horses infected with a strain that is less susceptible to IMD. Compound 1294 is safe in vivo in rodents and effective against $T$. gondii [30], C. parvum [32], P. falciparum [29] and B. bovis [33]. Importantly, it is nontoxic in mice following twice daily oral administration of $100 \mathrm{mg} / \mathrm{kg}$ for five days [29]. It has $91 \%$ oral bioavailability in rats and is likely cleared by hepatic metabolism [29]. In the current study, this compound demonstrated equal and substantial efficacy in vitro against both the TX isolate and the FL Exp variant 1 , which had an almost 15-fold difference in IMD susceptibility. Importantly, BKI 1294 resulted in complete parasite growth inhibition for both isolates, consistent with its novel mechanism of action. Although the $\mathrm{IC}_{50}$ of BKI 1294 was higher than that of IMD in vitro, its apicomplexan-selective target and its favorable pharmacokinetics and safety in other mammals [28-30] suggest that it could be useful for eliminating T. equi infection in horses. Further investigation of this class of compounds for this purpose is a focus of ongoing research.

Flow cytometry has been used to evaluate parasite infection of erythrocytes for multiple hemoprotozoan species, including B. bovis [50], Anaplasma marginale [51], B. gibsoni [52], B. canis [53], and P. berghei [54]. This technique relies on staining of infected erythrocyte populations with hydroethidine, which is taken up by live parasites within intact erythrocytes and converted to the fluorochrome ethidium bromide. Ethidium bromide intercalates into the DNA of viable parasites and can be utilized to differentiate infected erythrocytes from those that are uninfected or that contain non-viable parasites using flow cytometry [50]. It is therefore particularly relevant for assessment of drug susceptibility as only viable parasites are identified, in comparison to the traditional method of evaluating PPE by blood smear in which parasite viability cannot be determined. In this study, we were able to successfully apply this technique for $T$. equi-infected erythrocytes for the first time to evaluate parasite growth in drug susceptibility assays.

\section{Conclusions}

This study demonstrated variation in IMD susceptibility for the first time in vitro between two T. equi strains, with decreased susceptibility and incomplete parasite inhibition for the USDA FL strain. This finding was consistent with the inability of IMD to clear the same strain in vivo in four experimentally infected ponies, despite two rounds of treatment. Importantly, the emergence of IMD resistance during exposure to the drug was demonstrated in vitro. In contrast, no such in vitro variation in susceptibility between strains was observed for BKI compound 1294. This novel class of compounds may represent an effective alternative for the treatment of resistant T. equi infections in horses.

\section{Abbreviations}

BKI: Bumped kinase inhibitor; CDPK: Calcium-dependent protein kinase; FL: USDA Florida strain of T. equi; FL Exp variant 1: T. equi variant pulse exposed to IMD; FL Exp variant 2: T. equi variant continuously exposed to IMD; IC 50: 50\% inhibitory concentration; $I_{90}$ : 90\% inhibitory concentration; IMD: Imidocarb dipropionate; PPE: Percent parasitized erythrocytes; TX: novel T. equi isolate originating from Texas.

\section{Competing interests}

Wesley C. Van Voorhis is the President of ParaTheraTech Inc., a company which has the aim to market bumped kinase inhibitors for animal health. All other authors declare that they do not have any competing interests. 


\section{Authors' contributions}

$\mathrm{SH}$ designed and performed all drug assays, including obtaining and analyzing all flow cytometry data, initiated the TX isolate cultures, performed in vitro drug exposure of the parasites, conceived the study, and drafted the manuscript. JR treated the infected horses and performed all PCR assessments and follow up evaluation of the animals. LK provided the initial FL T. equi sample as well as substantial scientific guidance for parasite cultures. AL assisted with the design of the BKI drug assays and evaluation of $T$. equi CDPK, and provided data necessary for sequence alignment. KO provided scientific guidance for design of the BKI drug assays and analysis of CDPK genes. WV contributed scientific expertise regarding BKI compounds as well as providing compound 1294 for testing. DK critically revised the manuscript and contributed laboratory resources and personnel to support experimental procedures. RM conceived the study, performed sequence alignments, calculated $\mathrm{IC}_{50}$ and $\mathrm{IC}_{90}$ values, and critically revised the manuscript. All authors read and approved the final manuscript.

\section{Acknowledgements}

This work was supported in part by Zoetis-Morris Animal Foundation Fellowship Grant No. D12EQ-901 (SAH), USDA-NIFA-AFRI Fellowship Grant No. 2012-67011-19946 (JDR), the Grayson Jockey Club Research Foundation (RHM), and USDA-NIFA-AFRI Grant No. 2013-01149 (RHM).

Work in the Van Voorhis lab was supported by NIH grants 1 R01 Al089441, Al111341-01 and USDA grant \# 2014-06183.

The authors acknowledge Christina Macheres and Shelby Beckner for their valuable technical assistance, and Dr. Zhongsheng Zhang for initial synthesis of BKI 1294

\section{Author details}

'Department of Veterinary Microbiology and Pathology, College of Veterinary Medicine, Washington State University, Pullman, WA 99164-7040, USA. ${ }^{2}$ Animal Disease Research Unit, Agricultural Research Service, USDA, Pullman, WA 99164-6630, USA. ${ }^{3}$ Division of Allergy and Infectious Diseases and Center for Emerging and Re-emerging Infectious Diseases, School of Medicine, University of Washington, Seattle, WA 98109-4766, USA.

\section{Received: 9 September 2014 Accepted: 17 December 2014} Published online: 20 January 2015

\section{References}

1. Holbrook AA. Biology of equine piroplasmosis. J Am Vet Med Assoc. 1969;155:453-4.

2. Ribeiro IB, Camara AC, Bittencourt MV, Marcola TG, Paludo GR, Soto-Blanco B. Detection of Theileria equi in spleen and blood of asymptomatic piroplasm carrier horses. Acta Parasitol. 2013;58:218-22.

3. Ueti MW, Palmer GH, Kappmeyer LS, Statdfield M, Scoles GA, Knowles DP. Ability of the vector tick Boophilus microplus to acquire and transmit Babesia equi following feeding on chronically infected horses with low-level parasitemia. J Clin Microbiol. 2005;43:3755-9.

4. Ueti MW, Mealey RH, Kappmeyer LS, White SN, Kumpula-McWhirter N, Pelzel AM, et al. Re-emergence of the apicomplexan Theileria equi in the United States: elimination of persistent infection and transmission risk. PLoS One. 2012;7:e44713.

5. Committee on Infectious Diseases of Horses. Resolution \#21: Equine Piroplasmosis - Release of Test Negative Treated Horses. United States Animal Health Association 115th Annual Meeting. 2011. 11-1-2014.

6. Scoles GA, Hutcheson HJ, Schlater JL, Hennager SG, Pelzel AM, Knowles DP. Equine piroplasmosis associated with Amblyomma cajennense Ticks, Texas, USA. Emerg Infect Dis. 2011;17:1903-5.

7. Frerichs WM, Allen PC, Holbrook AA. Equine piroplasmosis (Babesia equi): therapeutic trials of imidocarb dihydrochloride in horses and donkeys. Vet Rec. 1973:93:73-5.

8. Kuttler KL, Zaugg JL, Gipson CA. Imidocarb and parvaquone in the treatment of piroplasmosis (Babesia equi) in equids. Am J Vet Res. 1987:48:1613-6.

9. Grause JF, Ueti MW, Nelson JT, Knowles DP, Kappmeyer LS, Bunn TO Efficacy of imidocarb dipropionate in eliminating Theileria equi from experimentally infected horses. Vet J. 2013;196:541-6.

10. Knowles Jr DP. Control of Babesia equi parasitemia. Parasitol Today. 1996;12:195-8.
11. Ding XC, Beck HP, Raso G. Plasmodium sensitivity to artemisinins: magic bullets hit elusive targets. Trends Parasitol. 2011;27:73-81.

12. Cohn LA, Birkenheuer AJ, Brunker JD, Ratcliff ER, Craig AW. Efficacy of atovaquone and azithromycin or imidocarb dipropionate in cats with acute cytauxzoonosis. J Vet Intern Med. 2011;25:55-60.

13. Lin EC, Chueh LL, Lin CN, Hsieh LE, Su BL. The therapeutic efficacy of two antibabesial strategies against Babesia gibsoni. Vet Parasitol. 2012;186:159-64.

14. Dahlstrom S, Aubouy A, Maiga-Ascofare O, Faucher JF, Wakpo A, Ezinmegnon $\mathrm{S}$, et al. Plasmodium falciparum Polymorphisms associated with ex vivo drug susceptibility and clinical effectiveness of artemisinin-based combination therapies in Benin. Antimicrob Agents Chemother. 2014;58:1-10.

15. Nagai A, Yokoyama N, Matsuo T, Bork S, Hirata H, Xuan X, et al. Growth-inhibitory effects of artesunate, pyrimethamine, and pamaquine against Babesia equi and Babesia caballi in in vitro cultures. Antimicrob Agents Chemother. 2003;47:800-3.

16. Wise LN, Ueti MW, Kappmeyer LS, Hines MT, White SN, Davis W, et al. In vitro activity of ponazuril against Theileria equi. Vet Parasitol. 2012;185:282-5.

17. Bork S, Yokoyama N, Matsuo T, Claveria FG, Fujisaki K, Igarashi I. Growth inhibitory effect of triclosan on equine and bovine Babesia parasites. Am J Trop Med Hyg. 2003:68:334-40.

18. Aboulaila M, Nakamura K, Govind Y, Yokoyama N, Igarashi I. Evaluation of the in vitro growth-inhibitory effect of epoxomicin on Babesia parasites. Vet Parasitol. 2010;167:19-27.

19. Munkhjargal T, Aboulaila M, Terkawi MA, Sivakumar T, Ichikawa M, Davaasuren $B$, et al. Inhibitory effects of pepstatin A and mefloquine on the growth of Babesia parasites. Am J Trop Med Hyg. 2012;87:681-8.

20. Salama AA, Aboulaila M, Terkawi MA, Mousa A, El-Sify A, Allaam M, et al. Inhibitory effect of allicin on the growth of Babesia and Theileria equi parasites. Parasitol Res. 2014;113:275-83.

21. Salama AA, Aboulaila M, Moussa AA, Nayel MA, El-Sify A, Terkawi MA, et al. Evaluation of in vitro and in vivo inhibitory effects of fusidic acid on Babesia and Theileria parasites. Vet Parasitol. 2013;191:1-10.

22. Rodriguez RI, Trees AJ. In vitro responsiveness of Babesia bovis to imidocarb dipropionate and the selection of a drug-adapted line. Vet Parasitol. 1996;62:35-41.

23. Preechapornkul $P$, Imwong $M$, Chotivanich $K$, Pongtavornpinyo $W$, Dondorp AM, Day NP, et al. Plasmodium falciparum pfmdr1 amplification, mefloquine resistance, and parasite fitness. Antimicrob Agents Chemother. 2009:53:1509-15.

24. Gaffar FR, Wilschut K, Franssen FF, de VE. An amino acid substitution in the Babesia bovis dihydrofolate reductase-thymidylate synthase gene is correlated to cross-resistance against pyrimethamine and WR99210. Mol Biochem Parasitol. 2004;133:209-19.

25. Dalgliesh RJ, Stewart NP. Tolerance to imidocarb induced experimentally in tick-transmitted Babesia argentina. Aust Vet J. 1977;53:176-80.

26. Doliwa C, Escotte-Binet S, Aubert D, Velard F, Schmid A, Geers R, et al. Induction of sulfadiazine resistance in vitro in Toxoplasma gondii. Exp Parasitol. 2013;133:131-6.

27. Hwang SJ, Yamasaki M, Nakamura K, Sasaki N, Murakami M, Wickramasekara Rajapakshage BK, et al. Development and characterization of a strain of Babesia gibsoni resistant to diminazene aceturate in vitro. J Vet Med Sci. 2010;72:765-71.

28. Ojo KK, Pfander C, Mueller NR, Burstroem C, Larson ET, Bryan CM, et al. Transmission of malaria to mosquitoes blocked by bumped kinase inhibitors. J Clin Invest. 2012;122:2301-5.

29. Ojo KK, Eastman RT, Vidadala R, Zhang Z, Rivas KL, Choi R, et al. A specific inhibitor of PfCDPK4 blocks malaria transmission: chemical-genetic validation. J Infect Dis. 2014;209:275-84.

30. Doggett JS, Ojo KK, Fan E, Maly DJ, Van Voorhis WC. Bumped Kinase Inhibitor 1294 Treats Established Toxoplasma gondii Infection. Antimicrob Agents Chemother. 2014:58:3547-9.

31. Murphy RC, Ojo KK, Larson ET, Castellanos-Gonzalez A, Perera BG, Keyloun KR, et al. Discovery of potent and selective inhibitors of Calcium-Dependent Protein Kinase 1 (CDPK1) from C. parvum and T. gondii. ACS Med Chem Lett. 2010;1:331-5.

32. Castellanos-Gonzalez A, White Jr AC, Ojo KK, Vidadala RS, Zhang Z, Reid MC, et al. A novel calcium-dependent protein kinase inhibitor as a lead compound for treating cryptosporidiosis. J Infect Dis. 2013;208:1342-8.

33. Keyloun KR, Reid MC, Choi R, Song Y, Fox AM, Hillesland HK, et al. The gatekeeper residue and beyond: homologous calcium-dependent protein 
kinases as drug development targets for veterinarian Apicomplexa parasites. Parasitology. 2014;141:1499-509.

34. Larson ET, Ojo KK, Murphy RC, Johnson SM, Zhang Z, Kim JE, et al. Multiple determinants for selective inhibition of apicomplexan calcium-dependent protein kinase CDPK1. J Med Chem. 2012;55:2803-10.

35. Johnson SM, Murphy RC, Geiger JA, DeRocher AE, Zhang Z, Ojo KK, et al. Development of Toxoplasma gondii calcium-dependent protein kinase 1 (TgCDPK1) inhibitors with potent anti-toxoplasma activity. J Med Chem. 2012;55:2416-26.

36. Knowles Jr DP, Perryman LE, Goff WL, Miller CD, Harrington RD, Gorham JR. A monoclonal antibody defines a geographically conserved surface protein epitope of Babesia equi merozoites. Infect Immun. 1991;59:2412-7.

37. Kappmeyer LS, Thiagarajan M, Herndon DR, Ramsay JD, Caler E, Djikeng A, et al. Comparative genomic analysis and phylogenetic position of Theileria equi. BMC Genomics. 2012;13:603.

38. Holman PJ, Chieves L, Frerichs WM, Olson D, Wagner GG. Babesia equi erythrocytic stage continuously cultured in an enriched medium. J Parasitol. 1994;80:232-6.

39. Belloli C, Crescenzo G, Lai O, Carofiglio V, Marang O, Ormas P. Pharmacokinetics of imidocarb dipropionate in horses after intramuscular administration. Equine Vet J. 2002;34:625-9.

40. Brasseur P, Lecoublet S, Kapel N, Favennec L, Ballet JJ. In vitro evaluation of drug susceptibilities of Babesia divergens isolates. Antimicrob Agents Chemother. 1998;42:818-20.

41. Naidoo V, Zweygarth E, Eloff JN, Swan GE. Identification of anti-babesial activity for four ethnoveterinary plants in vitro. Vet Parasitol. 2005;130:9-13.

42. Ramsay JD, Ueti MW, Johnson WC, Scoles GA, Knowles DP, Mealey RH. Lymphocytes and macrophages are infected by Theileria equi, but T cells and $B$ cells are not required to establish infection in vivo. PLOS One. 2013;8:e76996.

43. Knowles Jr DP, Perryman LE, Kappmeyer LS, Hennager SG. Detection of equine antibody to Babesia equi merozoite proteins by a monoclonal antibody-based competitive inhibition enzyme-linked immunosorbent assay. J Clin Microbiol. 1991;29:2056-8.

44. Nicolaiewsky TB, Richter MF, Lunge VR, Cunha CW, Delagostin O, Ikuta N et al. Detection of Babesia equi (Laveran, 1901) by nested polymerase chain reaction. Vet Parasitol. 2001;101:9-21.

45. Wang Z, Li X, Su D, Li Y, Wu L, Wang Y, et al. Residue depletion of imidocarb in Swine tissue. J Agric Food Chem. 2009;57:2324-8.

46. Lai O, Belloli C, Crescenzo G, Carofiglio V, Ormas P, Marangi O, et al. Depletion and bioavailability of imidocarb residues in sheep and goat tissues. Vet Hum Toxicol. 2002;44:79-83.

47. Aliu YO, Davis Jr RH, Camp BJ, Kuttler KL. Absorption, distribution, and excretion of imidocarb dipropionate in sheep. Am J Vet Res. 1977;38:2001-7.

48. Coldham NG, Moore AS, Dave M, Graham PJ, Sivapathasundaram S, Lake BG, et al. Imidocarb residues in edible bovine tissues and in vitro assessment of imidocarb metabolism and cytotoxicity. Drug Metab Dispos. 1995;23:501-5

49. Kerber CE, Ferreira F, Pereira MC. Control of equine piroplasmosis in Brazil. Onderstepoort J Vet Res. 1999;66:123-7.

50. Davis WC, Wyatt CR, Hamilton MJ, Goff WL. A rapid, reliable method of evaluating growth and viability of intraerythrocytic protozoan hemoparasites using fluorescence flow cytometry. Mem Inst Oswaldo Cruz. 1992;87 Suppl 3:235-9.

51. Coetzee JF, Apley MD, Kocan KM, Jones DE. Flow cytometric evaluation of selected antimicrobial efficacy for clearance of Anaplasma marginale in short-term erythrocyte cultures. J Vet Pharmacol Ther. 2006;29:173-83.

52. Fukata T, Ohnishi T, Okuda S, Sasai K, Baba E, Arakawa A. Detection of canine erythrocytes infected with Babesia gibsoni by flow cytometry. J Parasitol. 1996;82:641-2.

53. Bicalho KA, Ribeiro MF, Martins-Filho OA. Molecular fluorescent approach to assessing intraerythrocytic hemoprotozoan Babesia canis infection in dogs. Vet Parasitol. 2004;125:221-35.

54. Sobolewski P, Gramaglia I, Frangos JA, Intaglietta M, van der Heyde H. Plasmodium berghei resists killing by reactive oxygen species. Infect Immun. 2005;73:6704-10.

\section{Submit your next manuscript to BioMed Central and take full advantage of:}

- Convenient online submission

- Thorough peer review

- No space constraints or color figure charges

- Immediate publication on acceptance

- Inclusion in PubMed, CAS, Scopus and Google Scholar

- Research which is freely available for redistribution 